Supplement of

\title{
Investigation of structural changes of atmospheric aerosol samples during two thermal-optical measurement procedures (EUSAAR2, NIOSH870)
}

Theresa Haller et al.

Correspondence to: Theresa Haller (theresa.haller@univie.ac.at)

The copyright of individual parts of the supplement might differ from the article licence. 


\section{Supplementary Material}

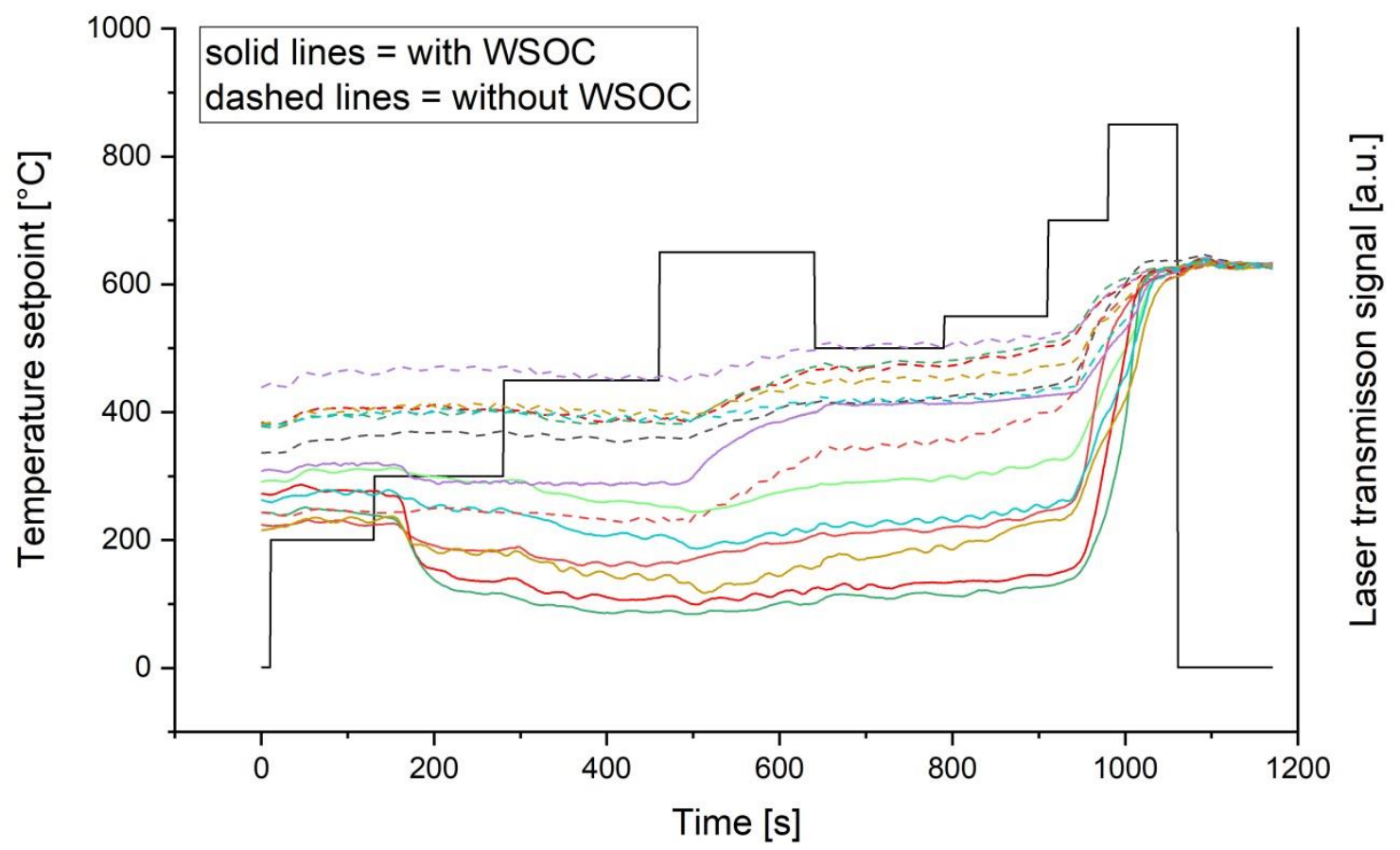

5 Figure S1: Progress of the laser signals for all samples with and without WSOC measured with the EUSAAR protocol. The lasersignals are normalized to the signal of the clean filter at the end of the procedure. 


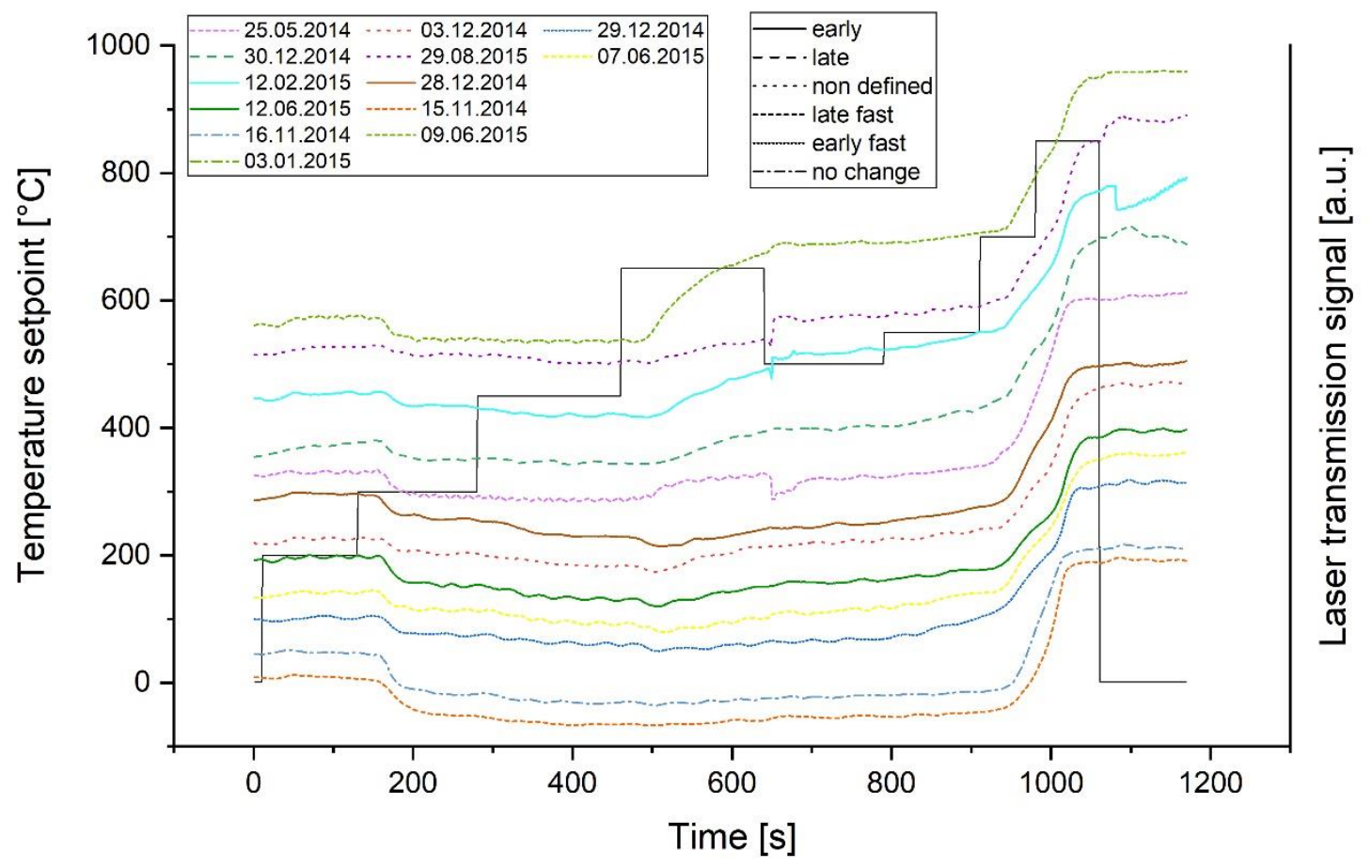

Figure S2: Transmission laser signals during the heating procedure of the EUSAAR2 protocol for all cases with a pronounced step 10 during the $300^{\circ} \mathrm{C}$ temperature level in He. The different line patterns represent the Raman categories early, late, late and fast, early and fast, no change and non-defined. The black solid line represents the ideal temperature curve in the instrument. 


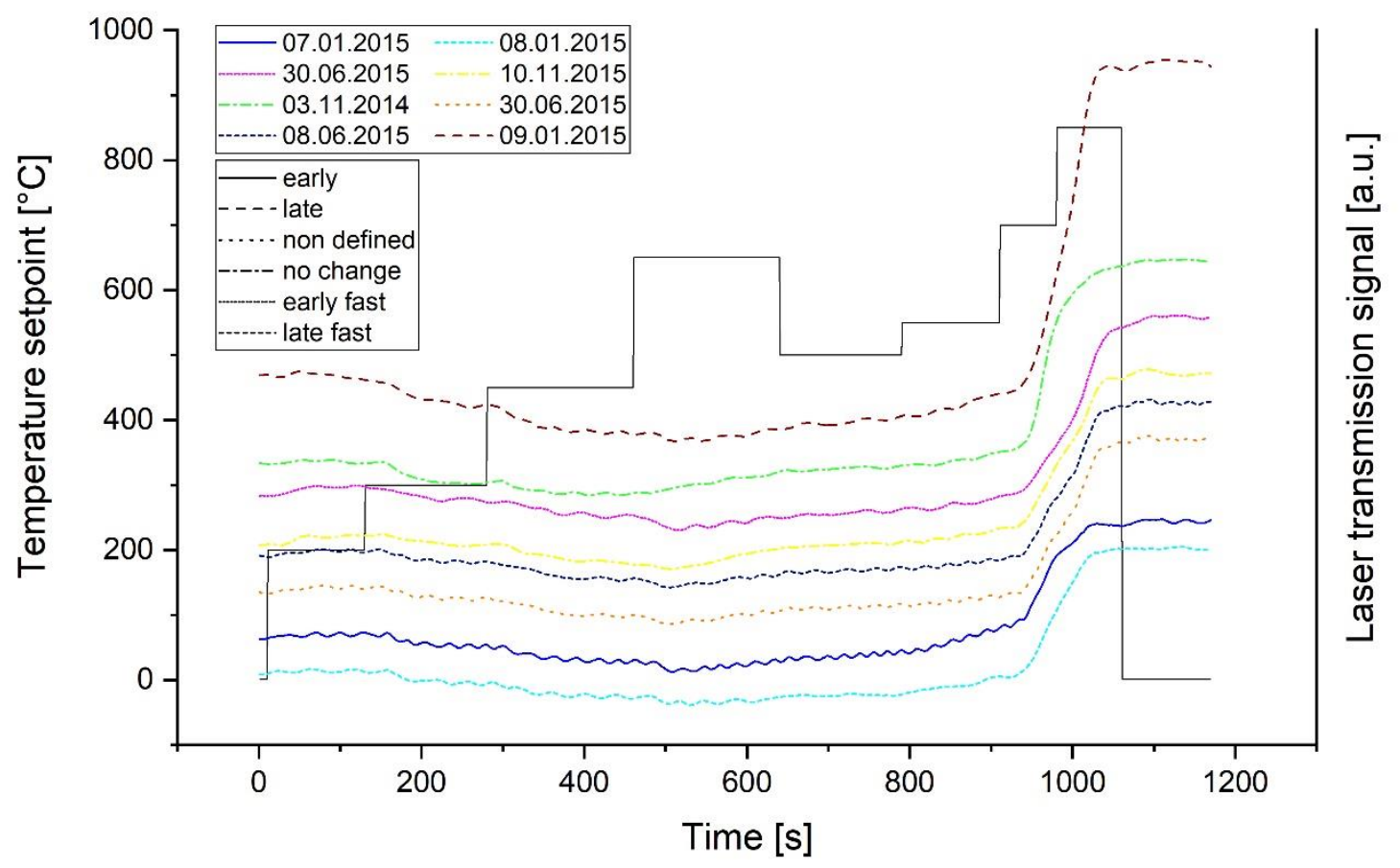

Figure S3: Transmission laser signals during the heating procedure of the EUSAAR2 protocol for all cases with a relatively steady decrease during the first three temperature levels in He. The different line patterns represent the Raman categories early, late, late and fast, early and fast, no change and non-defined. The black solid line represents the ideal temperature curve in the instrument. 


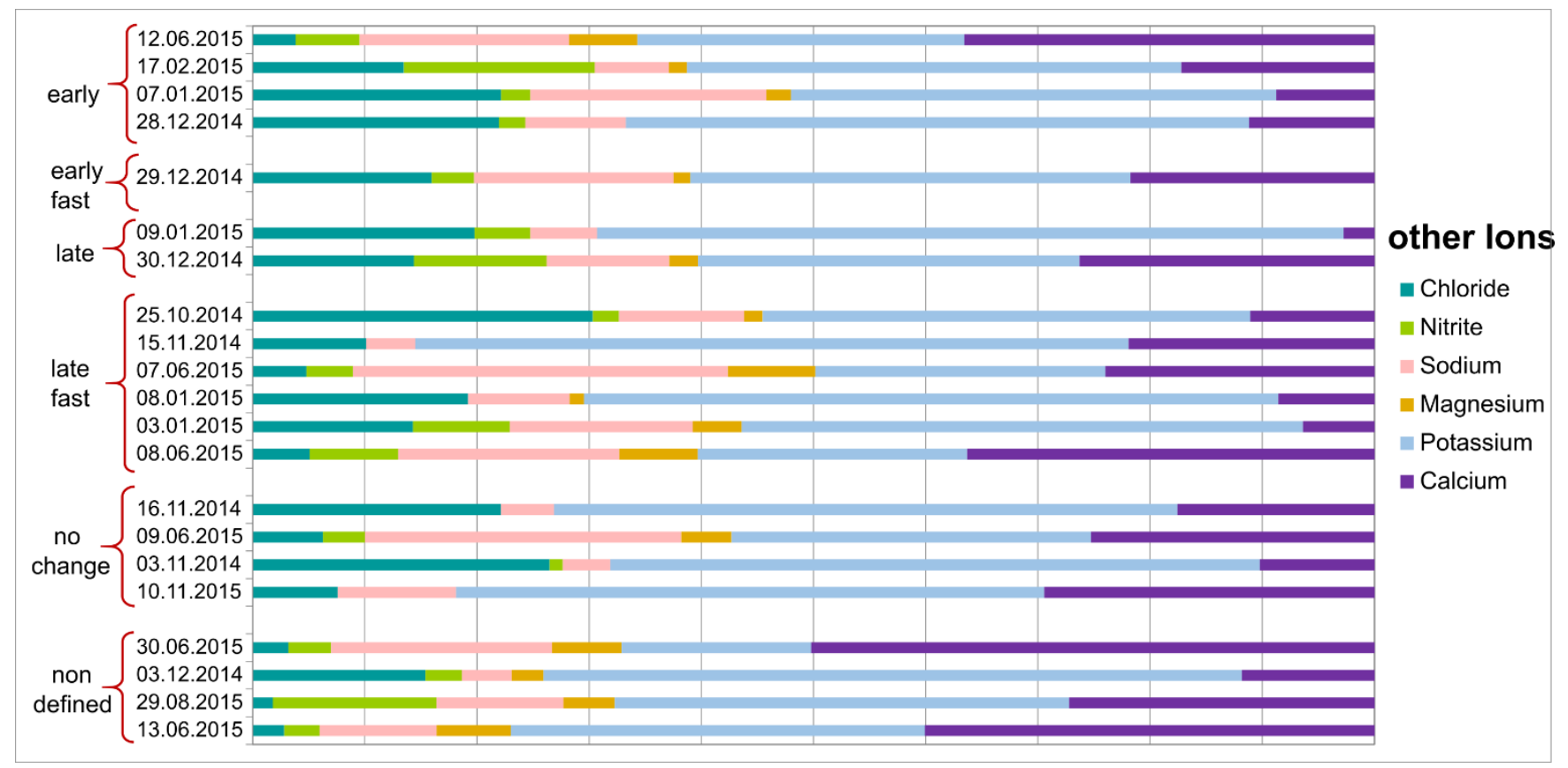

Figure S4: Fractions of analyzed minor ions, which were summarized as "other ions" in Fig. 7 in the main text, sorted by the categories representing the behavior of the Raman spectra during the thermal optical heating procedure. 